\section{Programm zur}

Qualitätssicherung

der Screening-

mammographie

Technische Aspekte

\author{
F. R. Verdun ${ }^{a}$, J.-P. De Landtsheer ${ }^{b}$, C. Jemelin ${ }^{c}$, B. Delanoy Ortega ${ }^{d}$, \\ C. Hessler ${ }^{e}$, J.-F. Valley ${ }^{a}$
}

a Institut de radiophysique appliquée, Lausanne

b Fondation pour le dépistage du cancer du sein, Lausanne

c Centre valaisan de dépistage, Sion

d Fondation genevoise pour le dépistage du cancer du sein, Genève

e Centre Hospitalier Universitaire Vaudois, Service de radiodiagnostic et de radiologie interventionnelle, Lausanne

\section{Zusammenfassung}

Gemäss Verordnung vom 23. Juni 1999 zur Qualitätssicherung der Programme für mammographisches Brustkrebsscreening sind Screeningkampagnen von den Krankenkassen zu finanzieren, sofern die angewendete Methode den Empfehlungen der Kommission der Europäischen Gemeinschaft [1] entspricht. Diese Empfehlungen betreffen nicht nur technische Anforderungen an die Bildgebungskette, sondern auch klinische Aspekte. Die vorliegende Arbeit befasst sich nur mit technischen Aspekten gemäss den Empfehlungen.

In den Kantonen Waadt, Wallis und Genf begannen 1999 organisierte Programme zum Screening auf Brustkrebs mittels Mammographie [2]. Im Verlauf des ersten Trimesters 1999 unterzog man rund zwanzig radiologische Anlagen einer Reihe nicht-invasiver Kontrollen, um ihre Konformität mit den Vorschriften zu überprüfen und sie dann zu den Programmen zuzulassen. Anlässlich dieser Kontrollen wurden zwei Einrichtungen von den Programmen ausgeschlossen, bei einem Zentrum wurde der Austausch einer FilmFolien-Kombination und bei sechs Einrichtungen die Intervention der Herstellerfirma verlangt.

Nach einer kurzen Beschreibung des Inhalts der für mammographisches Screening verlangten Kontrollen besprechen wir für die am Programm beteiligten Einrichtungen der drei genannten Westschweizer Kantone das Qualitätsniveau der Bildge-

Korrespondenz:

Dr. med. J.-P. De Landtsheer

Fondation pour le dépistage du cancer du sein

Rue du Bugnon 21

CH-1005 Lausanne bung und die an die Patientinnen abgegebene Strahlendosis. Wir werden unseren Beitrag mit einer allgemeinen kritischen Würdigung der im Rahmen der Kontrollen durchzuführenden Tests schliessen.

\section{Einführung}

Das Mammakarzinom ist in den industrialisierten Ländern eines der wichtigsten Probleme des öffentlichen Gesundheitswesens. In der Schweiz erfahren jedes Jahr nahezu viertausend Frauen, dass sie Brustkrebs haben und jährlich sterben daran mehr als eintausendvierhundert Frauen [3].

Zurzeit kennt man keine wirklich akzeptable Massnahme zur Primärprävention, die in der Lage wäre, das Risiko für Brustkrebs zu verringern. Die Mammographie ist jedoch die Untersuchung der Wahl, um das Mammakarzinom mit der best möglichen Effizienz zu entdecken. Sie ermöglicht nämlich den Nachweis der Frühzeichen eines Mammakarzinoms mehrere Jahre bevor klinische Symptome auftreten. Die Mammographie als Screening hat sich in den westlichen Ländern weitgehend als Methode der Wahl zur sekundären Prävention des Mammakarzinoms durchgesetzt [3].

Pilotprogramm im Kanton Waadt

1993 bis 1999 organisierte man in drei Bezirken des Kantons Waadt (Morges, Aubonne und Aigle) ein Pilotprogramm zum Screening auf Mammakarzinom mittels Mammographie. Im Rahmen dieses Programms erhielten alle in den betreffenden Bezirken wohnhaften über 50jährigen Frauen eine Einladung zur Mammographie (2 Strahlenrichtungen pro Brust) in Abständen von zwei Jahren. Je nach Wohnort erfolgten die kostenlosen Untersuchungen im Spital Aigle oder Morges. Diese Kampagne bezweckte, die geeignete Methodik vor ihrer allgemeinen Einführung im ganzen Kanton zu beurteilen. Aus diesem Anlass wurde die Stiftung «Fondation pour le dépistage du cancer du sein" (Stiftung für Mammakarzinomscreening) ins Leben gerufen, um die gesamte Logistik und Qualitätssicherung zu betreuen, die für den reibungslosen Ablauf der Operation notwendig sind: Einladen der Patientinnen, Verwaltung der Untersuchungstermine, Organisation von Doppel- oder sogar Dreifachbeurteilungen der Aufnahmen, Beurteilung der Leistung der Radiologen und der eingesetzten Einrichtungen. Das Institut für angewandte Radiophysik hatte in diesem Zusammenhang die Aufgabe, ein Programm zur Qualitätssicherung in den Einrichtungen zu erstellen, das mit den Empfehlungen der europäischen Union zu Screeninguntersuchungen vereinbar war. Jedes Jahr wurden beide am Versuchsprogramm beteiligten Einrichtungen gründlich überprüft und in jedem Trimester organisierte man eine Qualitätskontrolle der Bilder und der Strahlendosis. Ausserdem waren vor Ort die radiologischen Abteilungen verpflichtet, vor jedem Screeningsammeltermin die Entwicklung ihrer Filme zu kontrollieren. (Dieses Programm betraf 15000 Frauen, sein Deckungsbereich betrug ungefähr 70\%.) 


\section{Allgemeine Durchführung des Screening}

Nach dem Erlass des Bundesamts für Sozialversicherung vom 4. Juli 1997 gehört die Screeningmammographie zu den präventiven Leistungen zu Lasten der obligaten Krankenversicherung. Aufgrund dessen konnten die Kantone Genf und Wallis ihr Screeningprogramm einführen, während es im Kanton Waadt auf die gesamte Zielpopulation ausgedehnt wurde. Die drei Programme der französischsprachigen Schweiz sind nach denselben Grundprinzipien gestaltet und enthalten zahlreiche gemeinsame Aspekte. Einer dieser Aspekte betrifft die Organisation eines Netzwerks zur Durchführung der Screeningmammographien: in allen drei Kantonen kann jedes Institut für Radiologie am Programm teilnehmen, wenn es dies wünscht, gleichgültig ob es sich um eine private Praxis, eine Klinik oder ein Spital handelt. Bedingung ist lediglich, dass die Vorschriften des Programms erfüllt werden, insbesondere diejenigen zur Qualitätssicherung der für das Screening eingesetzten radiologischen Ausrüstung.

Am 23. Juni 1999 erliess der Bundesrat eine neue Verordnung über die Leistungen der Krankenversicherung. Darin werden die Bedingungen festgelegt, die erfüllt sein müssen, damit die im Rahmen organisierter Screeningprogramme durchgeführten Mammographien von den Krankenversicherungen der ganzen Schweiz finanziert werden. Insbesondere hält die Verordnung fest, dass die Kostenerstattung an die Beachtung der europäischen Richtlinien zur Qualitätskontrolle von Screeninguntersuchungen auf Mammakarzinom geknüpft ist.

\section{Ziele der Studie}

Es sollte überprüft werden, ob die Organisation der verschiedenen Programme zum Screening auf Mammakarzinom die europäischen Richtlinien erfüllte. Zudem galt es, mehrere Fragen $\mathrm{zu}$ beantworten, die sich aus der vereinbarten Funktionsweise ergaben:

- Sind die Institute für Radiologie, die sich am Screening beteiligen möchten, in der Lage, die notwendigen technischen und finanziellen Aufwendungen $\mathrm{zu}$ erbringen?

- Können radiologische Institute, in denen wenige Mammographien durchgeführt werden, das erforderliche Qualitätsniveau erreichen?

- Lässt sich eine wirksame Struktur aufbauen, um die Qualität jedes am Programm teilnehmenden radiologischen Instituts zu kontrollieren?

Die vorliegende Studie bezweckt, für alle am Screening beteiligten mammographischen Einrichtungen der Kantone Waadt und Wallis und für einige am Programm beteiligten Einrichtungen im Kanton Genf die Methode der Qualitätskontrolle und die Ergebnisse der Beurteilung vorzustellen. Sie ermöglicht auch eine erste Antwort auf Fragen zur Organisationsweise, welche aus verschiedenen Programmen zum Screening auf Mammakarzinom gewählt wurden.

\section{Organisation der Qualitätskontrolle}

In denjenigen europäischen Ländern, wo Screeninguntersuchungen organisiert werden, sind Medizinphysiker mit gründlichen Kontrollen jeder Bildgebungskette beauftragt. So soll ein optimales Verhältnis zwischen abgegebener Strahlendosis und Qualität der Aufnahme erreicht werden. Die Empfehlungen der europäischen Union dienen als Grundlage für Körperschaften, die auf lokaler oder nationaler Ebene ihre eigenen Forderungen definieren. Als das Versuchsprogramm im Kanton Waadt eingeführt wurde, gab es gesamtschweizerisch keine gesetzliche Grundlage zur Qualitätskontrolle der Einrichtungen für Mammographie. In Übereinstimmung mit der für das Programm verantwortlichen Stiftung entwarf das Institut für angewandte Strahlenphysik eine auf die europäischen Empfehlungen abgestützte Methodik. Dabei wurde jedoch die wöchentliche Qualitätskontrolle der Aufnahmen durch eine Qualitätskontrolle der Bilder und der Strahlendosis in dreimonatigen Abständen ersetzt, da die Anlagen nur an drei Halbtagen pro Woche, verteilt auf die Spitäler Aigle und Morges, für die Screeninguntersuchungen eingesetzt wurden.

Im übrigen hat das für die Strahlenschutzverordnung verantwortliche Bundesamt für Gesundheit (BAG) gewisse Anforderungen zur Qualitätssicherung an radiologischen Anlagen jeglicher Art herausgegeben. Beispielsweise werden detaillierte Kontrollen der Einrichtungen (sogenannte Abnahme- und Zustandsprüfung) nur anerkannt, wenn sie vom Personal der Firma durchgeführt werden, welche die Einrichtung verkaufte, oder von einem durch das BAG ermächtigten Elektroniker. Die Häufigkeit der Zustandsprüfungen radiologischer Einrichtungen ist auf dreijährige Intervalle festgelegt, ausser bei Anlagen für die Zahnheilkunde. Die Prüfungen müssen im Anschluss an eine Wartung erfolgen. Der Vorteil dieses Vorgehens ist, dass beim Aufdecken eines technischen Mangels dieser mit geringeren Kosten behoben werden kann. Ein Nachteil liegt allerdings darin, dass der Kunde keine Kontrolle über die Leistungen der Firma hat. Zudem würde dieses Vorgehen im Rahmen eines organisierten Screenings einen Leistungsvergleich der eingesetzten Anlagen erschweren.

Für mammographische Einrichtungen, die in einem Screeningprogramm eingesetzt werden, empfehlen die europäischen Richtlinien gründliche Kontrollen durch einen Diplomphysiker zweimal jährlich. In der Schweiz gibt es noch keine Vorschriften bezüglich der zur Kontrolle ermächtigten Personen. So kontrollierte ab dem 1. Januar 1999 das Institut für angewandte Strahlenphysik die Mehrzahl der am Screening in der Waadt beteiligten Einrichtungen, um ihre Übereinstimmung mit den Vorschriften sicherzustellen. Das Institut wurde auch mit der Prüfung einiger Einrichtungen im Wallis und im Kanton Genf beauftragt. Parallel dazu entwickelte man einen Kurs für Installateure radiologischer Einrichtungen und für das vom BAG autorisierte Wartungspersonal, damit sie künftig die Prüfungen durchführen können. 


\section{Abbildung 1}

Beschreibung des Testobjekts MTM 100 zur Ermittlung der Punktezahl für die Bildqualität.

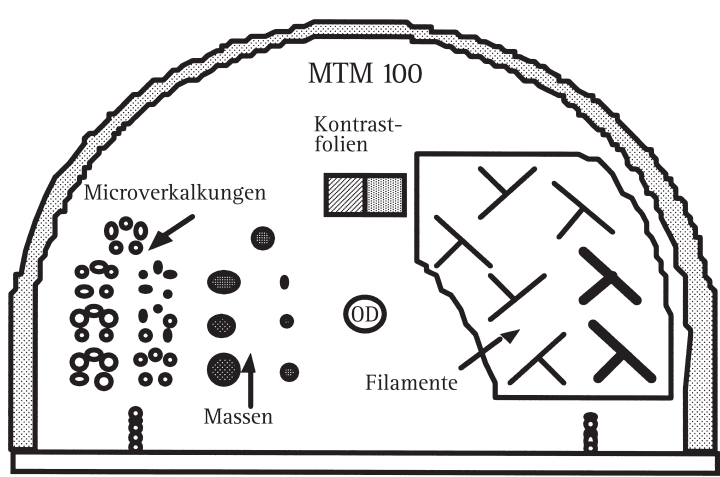

Es schien, als würde das BAG in Kürze verlangen, alle gründlichen Kontrollen von Screeningeinrichtungen hätten gemäss den geltenden Vorschriften für staatliche Kontrollen zu erfolgen. Bis heute hat aber das BAG keine derartige Vorschrift erlassen.

\section{Material und Methode}

Aufnahme der radiologischen Einheiten in das Programm

Vor der Aufnahme einer radiologischen Einheit in ein Screeningprogramm kontrollierte entweder das Institut für angewandte Strahlenphysik oder die Verkaufsfirma die ganze Bildgebungskette entsprechend den Empfehlungen der europäischen Gemeinschaft. Diese Prüfungen sind in der Literaturquelle [4] im Anhang im Einzelnen beschrieben.

Nach Zulassung durch die Stiftung mussten sich die radiologischen Einrichtungen zu einer Kontrolle der Filmentwicklung vor jedem Screeningsammeltermin und einer wöchentlichen Kontrolle der Bildqualität verpflichten. Angewendet wurde der in Abbildung 1 dargestellte Testobjekt MTM 100 oder ein anderes Testobjekt mit denselben Möglichkeiten. Die meisten Zentren entschieden sich für das Testobjekt MTM 100, was den Qualitätsvergleich der Aufnahmen erleichterte.

Das Testobjekt umfasst 7 Gruppen mit Mikroverkalkungen abnehmender Grösse, 7 Massen unterschiedlicher Grösse und 7 linearen, wenig kontrastgebenden Strukturen. Nach der Aufnahme dieses Objekts durch eine Röntgenanlage kann man eine Gesamtpunktezahl ermitteln, die als Indikator zur Beurteilung der Qualität der von der Anlage produzierten Bilder dient. Die Gesamtpunktezahl erhält man mit Hilfe der gegenüber Abbildung 1 dargestellten Tabelle durch Addition der Punkte, die jeder auf der Aufnahme dargestellten Struktur zugeordnet werden. Ausserdem erlaubt das Testobjekt die Bestimmung der Referenzschwärzungen und Messungen der Eintrittsdosis mit TLD-Dosimetern [5].

\begin{tabular}{|c|c|c|}
\hline Objektgruppe & $\begin{array}{c}\text { Punktezahl } \\
\text { bei vollständiger } \\
\text { Sichtbarkeit }\end{array}$ & $\begin{array}{c}\text { Punktezahl } \\
\text { bei teilweiser } \\
\text { Sichtbarkeit }\end{array}$ \\
\hline 1 & 1 & \\
2 & 2 & 3 \\
3 & 4 & 6 \\
4 & 8 & 12 \\
5 & 16 & 24 \\
6 & 32 & 48 \\
7 & 64 & \\
\hline
\end{tabular}

\section{Anhörung (Audit)}

Zur Qualitätssicherung der Bildgebungsketten der am Screeningprogramm in der Waadt und im Wallis beteiligten radiologischen Einrichtungen organisierten wir eine Anhörung zur Beurteilung des Zusammenhangs zwischen Expositionsdosis und Bildqualität. Die Anhörungstermine waren Anfang September 1999 in der Waadt und im April 2000 im Wallis. Auch in mehreren Genfer Zentren wurde diese Art Kontrolle im Lauf des Jahres 2000 durchgeführt. In jeder radiologischen Einrichtung wurde dasselbe mit thermolumineszenten Dosimetern ausgestattete Testobjekt MTM 100 automatisch exponiert. Zur Verbesserung der statistischen Aussagekraft wurden systematisch zwei Aufnahmen gemacht und jede Aufnahme von drei Experten beurteilt.

\section{Ergebnisse und Diskussion}

Abbildung 2 zeigt für alle in den drei Kantonen untersuchten radiologischen Einheiten die Punktezahlen und die Eintrittsdosen. Die Analyse der Ergebnisse zeigt, dass zur Zeit in den beurteilten Zentren die Bildqualität und die Dosis insgesamt zufriedenstellend sind. Für die im Rahmen des Screening applizierte Grenzdosis von 12 mGy beim Eintritt in eine Schichtdicke von $45 \mathrm{~mm}$ Brustdrüsenäquivalenz erreichen praktisch alle Radiologieeinheiten eine Bildqualitätpunktezahl über dem festgelegten Minimum. In Übereinstimmung mit Literaturquelle [5] beträgt die minimale Punktezahl 24 (siehe Abbildung 2).

Es ist allerdings zu berücksichtigen, dass die Zentren dieses Resultat nach Durchführung der Anpassungen erzielten, die auf Grund der detaillierten Kontrolle der Bildgebungskette gemäss Programmkoordination verlangt wurden. Zwei Einrichtungen erzielten bei den Kontrollen keine stabile Schwärzung nach Änderung der Dicke des Absorbers und hatten eine Bildqualität unter der von den Stiftungen festgelegten Grenze. Da die Anlagen alt waren, konnte kein Eingriff die Situation verbessern. Diese Anlagen 


\section{Abbildung 2}

Bilanz der Bildqualität - Dosis der radiologischen Einheiten.

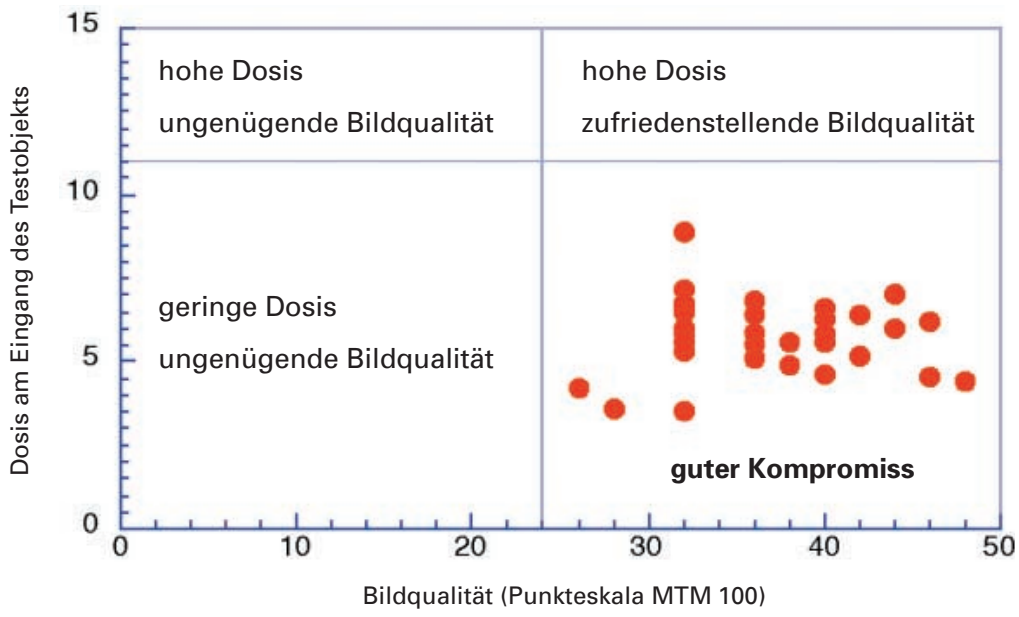

Abbildung 3

Schwärzungsgrad der Filme.

Von der Kommission der europäischen Gemeinschaft empfohlener Schwärzungsbereich

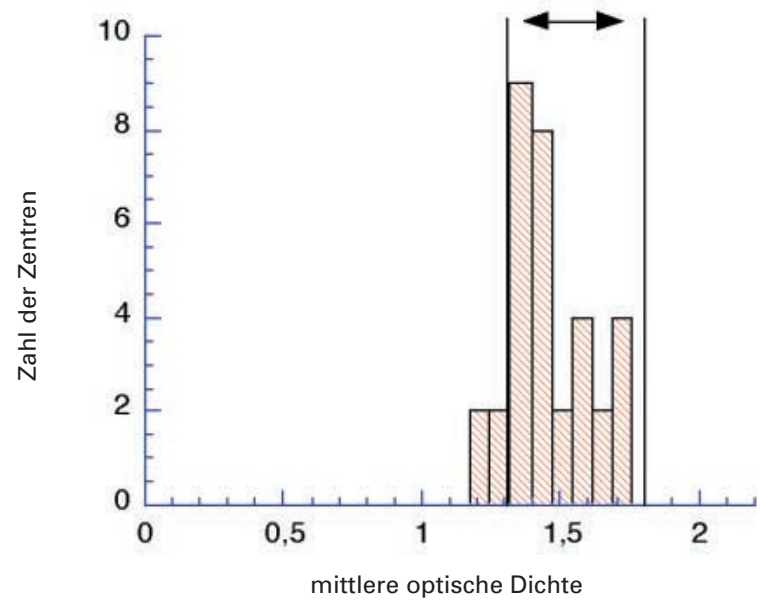

wurden durch moderne ersetzt, die nach Einstellen der Parameter die Anforderungen erfüllten.

In fünf radiologischen Einheiten musste die für die Wartung der Anlage verantwortliche Firma tätig werden, um die Regulierung der Zelle für automatische Exposition in Abhängigkeit von der Spannung zu justieren. Im Lauf der Zeit hatten diese radiologischen Einrichtungen den Bildschirm ausgewechselt, aber bei der Wartung der mammographischen Anlage hatte man es unterlassen, die erforderlichen Anpassungen vorzunehmen. Für die Installation war ein Eingriff der Firma in die Spannung-Dicke-Kurven notwendig, um die Anfertigung der Aufnahmen in einer vernünftigen Zeit zu ermöglichen. Schliesslich musste in einer radiologischen Einrichtung die etwa 10 Jahre alte Film-Folien-Kombination ausgetauscht werden.
Abbildung 3 zeigt das Basisschwärzungsniveau in den verschiedenen radiologischen Einrichtungen nach Detailkontrolle und Eichung durch die Firmen vor Zulassung zum Screeningprogramm. Bemerkenswert ist eine relativ starke Streuung im Bereich der verwendeten Schwärzungen, aber praktisch alle Zentren arbeiten heute im Rahmen der von der europäischen Union vorgeschlagenen Grenzen, nämlich einer Schwärzung zwischen 1,3 und 1,8. Diese Streuung kann auf den Mammogrammen in Abhängigkeit von der durch die medizinisch-technische Radiologieassistentin gewählten Positionierung der Zelle geringer sein. Mittelfristig sollte die Streuung verringert werden.

Bei der Kontrolle der Stabilität der Bildqualität liegt die verlangte minimale Punktezahl unterhalb derjenigen bei den jährlichen Kontrollen, denn die Aufnahmen werden im allgemeinen schneller und unter weniger günstigen Bedingungen beurteilt, als dies für die Beurteilung der Mammographien zu fordern ist. Derzeit wird verlangt, von jedem Objekt Mikroverkalkungen, Massen, Filamente - mindestens drei Gruppen zu entdecken. Diese Bedingung stellt sicher, dass die Bildverstärkerkette durchaus zufriedenstellende Aufnahmen produzieren kann.

\section{Schlussfolgerung}

Die hier vorgestellte Initiative bezweckte, die Homogenität der Mammographieanlagen zu beurteilen, die im Rahmen der Screeningprogramme in den Kantonen Waadt und Wallis eingesetzt werden. In Bezug auf das Genfer Screeningprogramm war die vergleichende Beurteilung eingeschränkt, weil die meisten Röntgenanlagen für Mammographie durch die für die Wartung verantwortlichen Firmen beurteilt worden waren.

Diese Beurteilung entsprach keiner reglementierten Forderung. Ziel war, die in anderen Ländern mit organisiertem Brustkrebsscreening angewandte Methodik der Qualitätskontrolle zu testen. Diese Art von Vorgehen bezweckt die Sicherstellung einer einheitlichen technischen Qualität, die unerlässlich ist, um Abweichungen zwischen verschiedenen Radiologen bei der Beurteilung derselben Aufnahmen zu verringern. Werden die radiologischen Anlagen durch verschiedene technische Dienste beurteilt, so kann man eine Information über die Einheitlichkeit der Leistungen nur durch eine Anhörung erhalten, die nach einheitlicher Methodik von externer Seite durchgeführt wird.

Die Qualitätssicherung im Bereich der herkömmlichen Radiologie (Röntgenaufnahmen, Durchleuchten, zahnmedizinisches Röntgen) ist durch die Anforderungen des BAG abgedeckt. Für die eben genannten radiologischen Untersuchungen wird eine alle drei Jahre von den Firmen auszuführende Wartung der Anlagen mit anschliessender Zustandskontrolle verlangt. Zudem muss der Betreiber der Anlage mindestens einmal jährlich die Stabilität kontrollieren lassen. Für die diagnostische oder screening- 
mässige Mammographie wird eine identische Methodik angewendet. Im übrigen empfiehlt die europäische Union Qualitätskontrollen der in einem Screeningprogramm eingesetzten Bildverstärkeranlagen in sechsmonatigen Abständen. In der Schweiz empfehlen wir diese Kontrollen einmal jährlich. Im Gegensatz zum üblichen Vorgehen hinsichtlich staatlicher Kontrollen scheint es uns nicht wünschenswert, die Kontrolle der für Screeningprogramme eingesetzten Röntgenanlagen für Mammographie an die Firmen zu delegieren. Die Installateure der Anlagen scheinen auch für die Kontrollen wenig motiviert zu sein, weil die Messungen andere Bestandteile der Bildgebungskette betreffen als die Installation der Mammographieanlage im engeren Sinn. Im übrigen erlaubt eine derartige Aufgabenverteilung keine objektive Kontrolle der von den Firmen durchgeführten Beurteilungen. In Ländern mit organisiertem Screening (USA, Kanada, skandinavische Länder, Niederlande, Grossbritannien und Frankreich) werden die Kontrollen von speziell ausgebildeten Medizinphysikern durchgeführt. Das BAG erlaubt, diese Strategie auch in der Schweiz zu übernehmen.

Man könnte demnach für die Screeningmammographie eine Strategie auf zwei Ebenen anwenden: Wartung und Zustandskontrolle alle drei Jahre unter der Zuständigkeit der Firmen, jährliche Qualitätskontrolle der Bildgebungskette durch Medizinphysiker. Es ist zu hoffen, dass das BAG bei der Formulierung der Richtlinien zur Qualitätskontrolle im Bereich der Screeningmammographie diese Argumente berücksichtigt.

Die Einführung der Screeningmammographie mit ihren qualitativen Anforderungen hat eine deutlich verbesserte Qualität der Aufnahmen ermöglicht. Alle in der Waadt und im Wallis am Programm beteiligten Bildgebungsketten entsprechen derzeit den europäischen Empfehlungen.

Für die am Programm im Kanton Genf beteiligten Einrichtungen könnte nur eine Anhörung in allen Zentren eine Konformität mit den Empfehlungen sicherstellen. Alle Zentren stimmten den vom Institut für angewandte Radiologie vorgeschlagenen technischen Änderungen zu, wenn immer solche nötig waren. Erwähnenswert ist allerdings, dass trotz der von gewissen Firmen durchgeführten Kontrolle die Film-Folien-Kombination in zwei Zentren nicht den Empfehlungen entsprach.
Auf die an der Durchführung der Screeningmammographien beteiligten Personen warten noch weitere Herausforderungen. Es steht heute fest, dass die Mammographieanlagen die Voraussetzungen für die Produktion qualitativ guter Aufnahmen erfüllen, doch gibt es weitere Mittel, um die Bildqualität ohne Erhöhung der Strahlendosis zu verbessern. Die Mammographie ist nämlich ein bildgebendes Verfahren, das nicht nur eine ausgezeichnete Bildgebungskette bedingt, sondern auch gezielt ausgebildete technische Assistentinnen und Assistenten. Sowohl die Lagerung der Mamma als auch ihre Kompression sind Schlüsselfaktoren für das Gelingen der Aufnahmen, wie dies Basset et al. [6] belegen.

Diese Forderungen sind umso grundlegender, als es sich um Screeningprogramme handelt. Wenn die Radiologen entscheiden müssen, ob ergänzende Untersuchungen angezeigt sind, stehen ihnen als Entscheidungsgrundlage nur die mammographischen Aufnahmen zur Verfügung.

\section{Literatur}

1 De Wolf CJM, Perry NM (eds). European guidelines for quality assurance in mammography screening. $2^{\text {nd }}$ edition. Luxemburg: European Commission; 1996.

2 De Landtsheer J-P, Delanoy Ortega B, Jemelin C. Dépistage du cancer du sein: analyse comparée de trois programmes suisses. Med Hyg 2000;2306:1407-10.

3 De Landtsheer J-P, Hessler C, Levi F, Delaloye J-F, De Grandi P, Paccaud F. Dépistage organisé du cancer du sein et pratique médicale. L'expérience vaudoise: bilan et perspectives. Bull Med Suisses 1998;79(34):1615-9.

4 Procédure concernant le contrôle de qualité des installations de mammographie impliquées dans programme pilote de dépistage du cancer du sein. Lausanne: Institut de Radiophysique Appliquée; 1998.

5 Noël A, Stines J, Zerhouni M. Développement d'un fantôme en matériau équivalent-sein pour la mammographie. Revue Européenne de Biotechnologie Médicale 1993;15(7):421-3.

6 Bassett LW, Farria DM, Bansal S, Farquhar MA, Wilcox PA, Feig SA. Reasons for failure of a mammography unit at clinical image review in the American College of Radiology Mammography Accreditation Program. Radiology 2000; 215:698-702.

La version française a paru dans le no 12/2001 